\title{
Evaluación de los músculos respiratorios en la parálisis diafragmática bilateral
}

\author{
CATALINA BRICEÑO V.*, TOMÁS REYES B.**, \\ JOSEFINA SÁEZ B.** y FERNANDO SALDÍAS P.*
}

\section{Assessment of respiratory muscle strength in bilateral diaphragmatic paralysis}

Measurement of respiratory muscle strength is useful in order to detect respiratory muscle weakness and to quantify its severity. Apropos of a patient with bilateral diaphragmatic paralysis, we review the clinical manifestations and methods for assessing the strength of the respiratory muscles. In patients with severe respiratory muscle weakness, vital capacity and total lung capacity are reduced but are a non-specific and relatively insensitive measure. Conventionally, inspiratory and expiratory muscle strength has been assessed by maximal inspiratory and expiratory mouth pressures sustained for one second (PIMax and PEMax). The sniff manoeuvre is natural and probably easier to perform. Sniff pressures are more reproducible and useful measure of diaphragmatic strength. However, the PIMaxPEMax and sniff manoeuvres are volition dependent, and submaximal efforts are most likely to occur in patients who are ill or breathless. Non-volitional tests include measurements of twitch esophageal, gastric and transdiaphragmatic pressure during bilateral electrical and magnetic phrenic nerve stimulation. Electrical phrenic nerve stimulation is technically difficult and is also uncomfortable and painful. Magnetic phrenic nerve stimulation is less painful and transdiaphragmatic pressure is reproducible in normal subjects. Systematic clinical evaluation and additional laboratory tests allow the diagnosis in most patients with respiratory muscle weakness.

Key words: Respiratory muscle strength, diaphragmatic function, diaphragm paralysis, lung volumes, phrenic nerve stimulation.

\section{Resumen}

La evaluación de la fuerza de los músculos respiratorios permite diagnosticar y cuantificar la gravedad de la debilidad muscular en diferentes enfermedades. A propósito de un paciente con parálisis diafragmática bilateral, hemos revisado el cuadro clínico y los procedimientos diagnósticos para evaluar la fuerza de los músculos respiratorios. En los pacientes con debilidad muscular respiratoria severa, disminuye la capacidad vital y la capacidad pulmonar total, pero es una medida inespecifica y relativamente insensible. Tradicionalmente, la fuerza muscular respiratoria es evaluada midiendo la presión inspiratoria y espiratoria máximas en la boca sostenidas durante un segundo (PIMax y PEMax). La medición de la presión inspiratoria máxima en la nariz (SNIP) es una maniobra natural, más simple de medir y más reproducible, siendo útil en la evaluación de la fuerza diafragmática. Sin embargo, estas técnicas no invasivas son operador dependiente, por lo tanto, esfuerzos submáximos es más probable que ocurran en pacientes graves o con disnea. Las mediciones de las presiones esofágica, gástrica y transdiafragmática mediante estimulación eléctrica o magnética del nervio frénico no son dependientes de la voluntad y son más confiables. Sin embargo, la estimulación eléctrica del nervio frénico es técnicamente difícil y puede ser incómoda y dolorosa. La estimulación magnética del nervio frénico es menos dolorosa y la medición de la presión transdiafragmática es reproducible en sujetos normales. La evaluación clínica sistemática y los exámenes de laboratorio complementarios permiten establecer el diagnóstico en la mayoría de los pacientes con debilidad de los músculos respiratorios.

Palabras clave: Fuerza de los músculos respiratorios, función diafragmática, parálisis diafragmática, volúmenes pulmonares, estimulación del nervio frénico.

* Departamento de Enfermedades Respiratorias, Facultad de Medicina, Pontificia Universidad Católica de Chile, Santiago de Chile.

** Internos de Medicina, Facultad de Medicina, Pontificia Universidad Católica de Chile. 


\section{Introducción}

El compromiso funcional de los músculos respiratorios suele afectar a pacientes con enfermedades agudas y crónicas de diferentes sistemas $\mathrm{u}$ órganos, a menudo no es detectado ni tratado en forma apropiada, ocasionando morbilidad y mortalidad significativas ${ }^{1}$ (Tabla 1). La debilidad de los músculos respiratorios es un factor agravante en múltiples condiciones, tales como la enfermedad pulmonar obstructiva crónica (EPOC), insuficiencia cardiaca congestiva, malnutrición, enfermedades neuromusculares, endocrinas y metabólicas, vasculitis y $\operatorname{sepsis}^{2-6}$. La medición de la fuerza de los músculos respiratorios permite establecer el diagnóstico, cuantificar la gravedad de la disfunción y planificar el tratamiento a largo plazo $^{1}$. En la Tabla 2 se describen las principales indicaciones de la evaluación de la función muscular respiratoria.

La parálisis diafragmática se caracteriza por una pérdida completa de la movilidad del diafragma, con ascenso diafragmático secundario, debido a aplasia o atrofia de las fibras musculares o una lesión del nervio frénico, sin un defecto de su continuidad ${ }^{1,7,8}$. En adultos, la principal causa es el trauma de columna cervical y las enfermedades neuromusculares: miastenia gravis, enfermedades del asta anterior, distrofias musculares y miopatías $^{7,8}$. Se puede observar en $2-20 \%$ de los pacientes sometidos a cirugía cardiaca y ocasionalmente debido a invasión tumoral. Su incidencia es desconocida debido a que frecuentemente no es diagnosticada ${ }^{7,8}$. Los pacientes suelen consultar por disnea de esfuerzos progresiva, que se agrava con los cambios de posición, al agacharse o acostarse, siendo con frecuencia invalidante, lo cual sugiere el diagnóstico ${ }^{1}$. En relación a un caso clínico, examinaremos los principales elementos diagnósticos de esta entidad.

\section{Caso clínico}

Hombre de 69 años, ex-fumador de 50 paquetes/año, no fuma hace 17 años, procedente de
Tabla 1. Enfermedades asociadas a disfunción muscular respiratoria

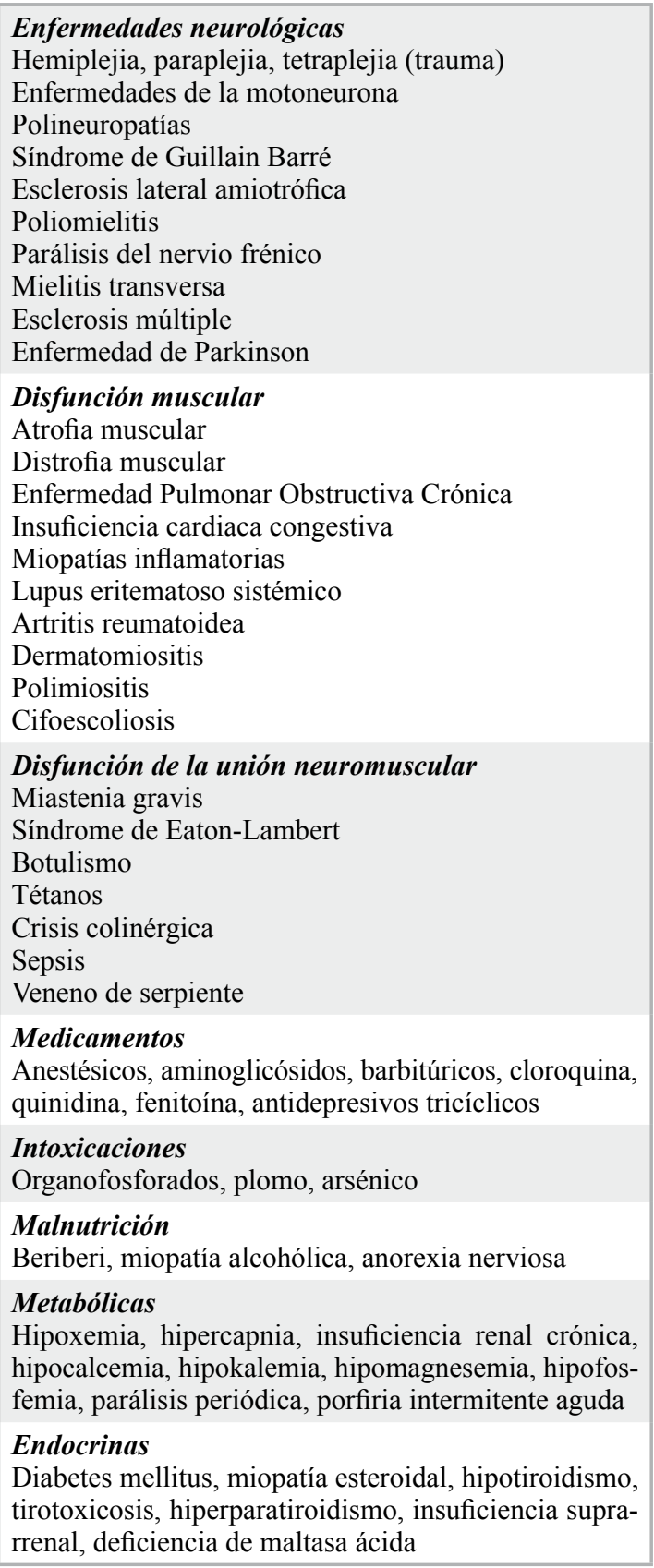

Tabla 2. Principales indicaciones para la medición de la fuerza de los músculos respiratorios

\footnotetext{
Evaluación de pacientes con disnea y tos de origen incierto

Evaluación funcional respiratoria en pacientes con enfermedades neuromusculares

Evaluación de la fuerza de los músculos respiratorios en pacientes con enfermedades cardiopulmonares

Evaluación del paciente crítico y protocolos de desconexión de ventilación mecánica (weaning)

Evaluación de intervenciones terapéuticas (seguimiento de pacientes con enfermedades cardiovasculares, neuromusculares o respiratorias crónicas, pacientes en terapia esteroidal, pacientes en entrenamiento muscular inspiratorio)
} 
Tabla 3. Exámenes de laboratorio de un paciente adulto con parálisis diafragmática bilateral

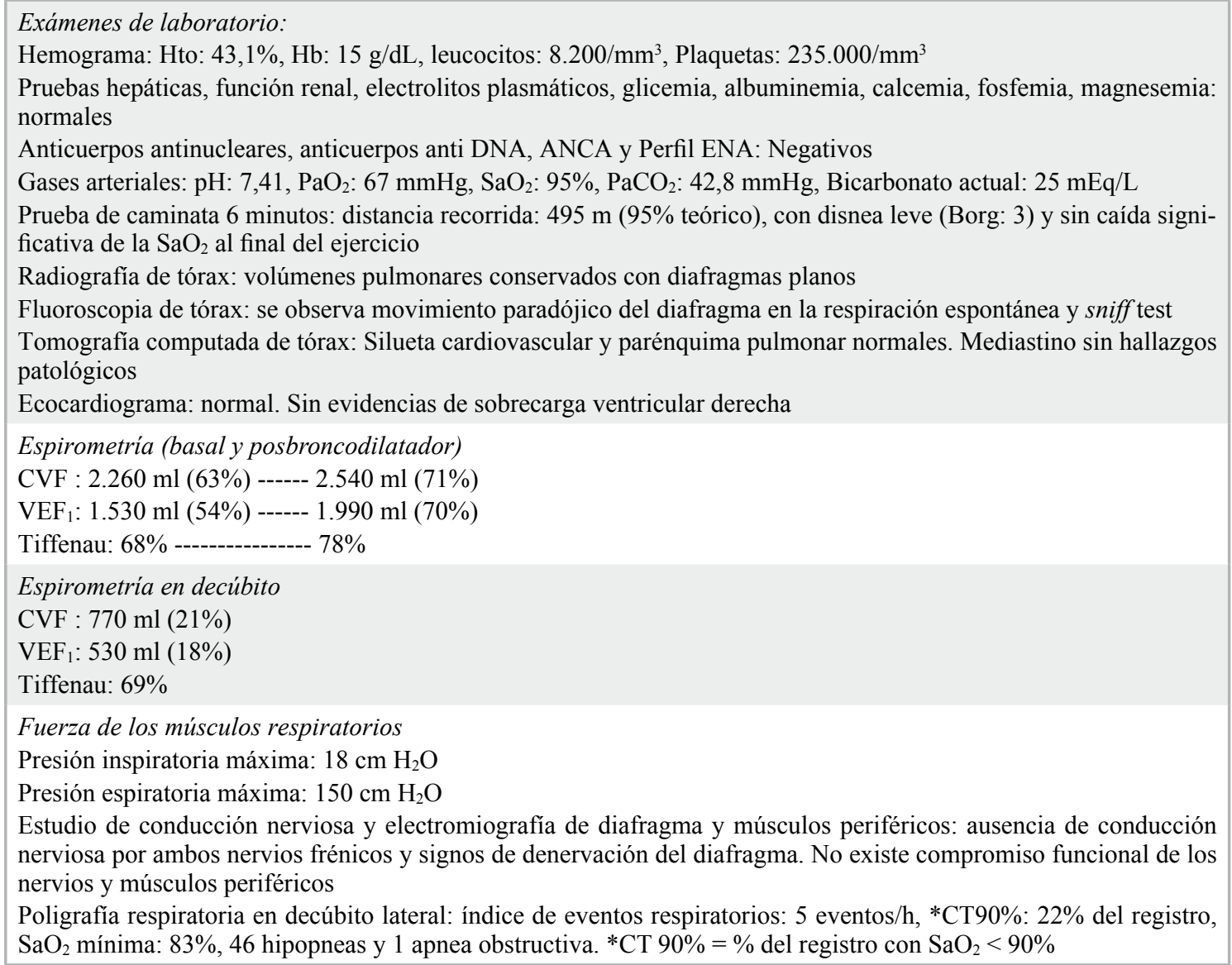

Coquimbo, portador de cardiopatía coronaria, antecedente de cirugía de revascularización miocárdica (triple bypass aortocoronario) en 2011, con dehiscencia esternal postoperatoria y complicaciones respiratorias postoperatorias. Consulta por disnea de medianos esfuerzos que se acentúa en decúbito dorsal y al nadar en el mar, y ortopnea de cinco almohadas desde que se operó en el 2011. En el examen físico destaca: FC: 72 lat/min, PA: 120/70 mmHg, FR: $14 \mathrm{resp} / \mathrm{min}$, $\mathrm{T}^{\mathrm{o}}: 37^{\circ} \mathrm{C}, \mathrm{SaO}_{2}: 96 \%$ respirando aire ambiente. Peso: $92 \mathrm{~kg}$. Talla: $1,73 \mathrm{~m}$. IMC: $30,7 \mathrm{~kg} / \mathrm{m}^{2}$. Perímetro cervical: $47 \mathrm{~cm}$. Escala de Mallampati: Clase I. Examen pulmonar: sonoridad normal, MP conservado sin ruidos agregados. Respiración paradójica y uso de músculos respiratorios accesorios. No tolera la posición en decúbito dorsal. En la Tabla 3 se describen los principales hallazgos de los exámenes de laboratorio, los cuales permitieron confirmar el diagnóstico de parálisis diafragmática bilateral postoperatoria de cirugía de revascularización miocárdica, cuantificar su gravedad y planificar el tratamiento a largo plazo. Las principales recomendaciones indicadas al paciente fueron bajar de peso, dormir en posición semisentada, uso de BiPAP nasal nocturno, realizar ejercicios de músculos respiratorios con válvula umbral, prevención de infecciones respiratorias y optimización del manejo de las comorbilidades.

\section{Discusión}

La evaluación funcional de los músculos respiratorios comprende la evaluación clínica (historia y examen físico) inicial, la medición de los volúmenes pulmonares, intercambio gaseoso y fuerza de los músculos respiratorios, los estudios de imágenes estáticos y dinámicos, la evaluación cardiovascular y la poligrafía respiratoria o polisomnografía ${ }^{1,9}$.

La parálisis diafragmática unilateral puede ser asintomática u ocasionar disnea en presencia de 
infecciones respiratorias intercurrentes o aumento de la demanda ventilatoria ${ }^{7,8}$. Los pacientes con parálisis diafragmática bilateral suelen consultar por disnea de esfuerzos progresiva que en los casos más graves puede ser de reposo, la cual se agrava al agacharse o en decúbito supino, no pueden permanecer acostados, refieren ortopnea y aumento de la disnea al bañarse en piscina o el mar. La ortopnea obligada y la respiración paradójica o alternante aparecen cuando la fuerza del diafragma disminuye a $25 \%$ del valor normal ${ }^{10}$. En posición sentada, los músculos abdominales pueden ser reclutados durante la espiración, lo cual eleva el diafragma y facilita su descenso por efecto de la gravedad durante la inspiración. La falla ventilatoria debido a la debilidad de los músculos respiratorios se desarrolla gradualmente y la hipoventilación suele aparecer primero durante el sueño ${ }^{11}$. Los casos más avanzados evolucionan con insuficiencia respiratoria global, hipertensión pulmonar y corazón pulmonar crónico.

\section{Estudios de imágenes}

La evaluación radiológica de la función del diafragma tradicionalmente se ha realizado con la radiografía y fluoroscopia de tórax en posición erecta $^{1}$. En la parálisis diafragmática unilateral es posible observar la elevación del hemidiafragma y en la parálisis bilateral se puede objetivar reducción del volumen pulmonar y elevación de ambos domos del diafragma ${ }^{12}$. La parálisis diafragmática también podría ser detectada en la fluoroscopia con el paciente en posición supina realizando maniobras de olfateo breves y agudas ${ }^{1}$. En condiciones normales, el hemidiafragma izquierdo se mueve más que el derecho, con excursión craniocaudal de 6 y $3 \mathrm{~cm}$ respectivamente. Los movimientos paradójicos aparecen cuando la presión ejercida por el abdomen supera la fuerza del diafragma, por lo cual el movimiento se invierte durante la inspiración y las maniobras de olfateo. Esto puede ocurrir en la parálisis diafragmática debido a lesión o compromiso tumoral de los nervios frénicos. El ultrasonido en tiempo real durante la respiración espontánea permite diferenciar la parálisis diafragmática de una masa abdominal ${ }^{13,14}$. Este examen sería de utilidad en la evaluación de la parálisis diafragmática, ruptura diafragmática, anomalías de la caja torácica y defectos abdominales en el recién nacido.

\section{Evaluación funcional respiratoria}

La debilidad de los músculos inspiratorios y espiratorios de magnitud moderada y severa reduce la capacidad vital (CV), por lo tanto, la capacidad vital normal permite descartar disfun- ción significativa de los músculos respiratorios ${ }^{15}$. La medición de la capacidad vital es dependiente del esfuerzo y la cooperación del paciente, está disminuida en múltiples afecciones cardiovasculares, metabólicas y sistémicas. Por lo tanto, la medición de la $\mathrm{CV}$ es poco sensible y específica para la pesquisa de disfunción de los músculos respiratorios $^{1,26}$. En pacientes con parálisis diafragmática, la reducción de la capacidad vital aumenta en decúbito, especialmente en pacientes con ortopnea y respiración paradójica ${ }^{10}$.

El compromiso funcional de los músculos inspiratorios se acompaña de reducción de la capacidad pulmonar total (CPT) y capacidad residual funcional (CRF), conservándose el volumen residual ${ }^{1,10}$. La resistencia de la vía aérea es normal. La capacidad de difusión $\left(\mathrm{DL}_{\mathrm{CO}}\right)$ está disminuida pero es normal cuando se ajusta al volumen $\left(\mathrm{DL}_{\mathrm{CO}} / \mathrm{VA}\right)^{1}$.

La parálisis diafragmática bilateral aislada rara vez ocasiona insuficiencia ventilatoria ${ }^{16}$. Inicialmente, la hipoxemia e hipercapnia se desarrollan durante el sueño, lo cual puede ser detectado mediante una oximetría nocturna, poligrafía respiratoria o polisomnografía. En algunos pacientes con disfunción diafragmática severa puede haber desaturación arterial durante el sueño, lo cual puede ser agravado por el colapso de la vía aérea superior durante el sueño, especialmente en pacientes con enfermedades neuromusculares crónicas ${ }^{11}$. Desde el punto de vista clínico, es importante destacar que la presencia de hipercapnia diurna traduce debilidad muscular severa (inferior al 30\% del teórico) con un pobre pronóstico funcional y vital ${ }^{17}$

\section{Evaluación de la fuerza de los músculos respiratorios}

La medición de la presión inspiratoria máxima (PIMax) y presión espiratoria máxima (PEMax) en la boca permite estimar la fuerza global de los músculos respiratorios ${ }^{18-20}$. Es un examen no invasivo, reproducible, de costo razonable, tiene valores de referencia normales en niños y adultos, depende de la edad, género, posición, volumen pulmonar y características de la pieza bucal $^{18-21}$. Valores elevados de PIMax $(80 \mathrm{~cm}$ $\left.\mathrm{H}_{2} \mathrm{O}\right)$ y PEMax $\left(90 \mathrm{~cm} \mathrm{H}_{2} \mathrm{O}\right)$ permiten excluir una disfunción significativa de los músculos respiratorios $^{18-20}$. La PIMax es medida desde volumen residual y la PEMax es medida desde capacidad pulmonar total, en posición sentada con una pieza bucal estándar y pinza nasal. Se consigna el valor más elevado que se sostiene por un segundo. Los equipos portátiles permiten evaluar la fuerza de los músculos respiratorios al lado de la cama del 
paciente, especialmente en los enfermos críticos en ventilación mecánica o durante la fase de desconexión del ventilador ${ }^{22}$. Las principales desventajas de esta medición es ser operador dependiente, la maniobra es demandante y ocasiona incomodidad, depende de la motivación del paciente, tiene curva de aprendizaje, el manejo de las fugas alrededor de la boquilla especialmente en pacientes ancianos o con deterioro cognitivo, se ha observado una amplia variabilidad entre individuos y rango de valores, incluso en población normal ${ }^{23}$.

La medición de la presión nasal en una maniobra de olfateo máxima (SNIP) permite estimar la fuerza de los músculos inspiratorios con mayor fiabilidad y menos variabilidad que la medición de la PIMax ${ }^{24,25}$. Esta maniobra se mide desde capacidad residual funcional porque la fuerza muscular inspiratoria es sobrestimada a volúmenes pulmonares inferiores a la CRF debido a la presión de retracción elástica del tórax ${ }^{26}$. Los valores normales en varones son mayores de 70 $\mathrm{cm} \mathrm{H}_{2} \mathrm{O}$ y en mujeres mayores de $60 \mathrm{~cm} \mathrm{H}_{2} \mathrm{O}^{27}$. Las principales desventajas de la medición de SNIP es ser operador dependiente, depende de la voluntad y motivación del paciente, esfuerzos submáximos son más frecuentes en enfermos graves o con disnea severa, la obstrucción nasal anatómica o funcional (rinitis, pólipos, desviación septal) puede afectar la transmisión de la presión desde el rinofarinx, las enfermedades pulmonares obstructivas y restrictivas pueden disminuir la transmisión de la presión pleural a la vía aérea ${ }^{15,24,28}$. Sin embargo, esta medición es muy útil en la evaluación de pacientes con enfermedades neuromusculares, incluyendo la parálisis diafragmática.

La evaluación de la fuerza de los músculos inspiratorios mediante la PIMax y SNIP son complementarias y constituyen los principales procedimientos no invasivos empleados para diagnosticar y cuantificar la gravedad de la debilidad de los músculos respiratorios.

En algunos pacientes con deterioro cognitivo, disnea o limitación funcional severa no es posible objetivar la debilidad de los músculos respiratorios mediante procedimientos no invasivos, en estos casos se puede medir la presión esofágica, gástrica y transdiafragmática (Pdi) mediante la instalación de balones bajo anestesia local en el tercio medio del esófago y en el cuerpo del estómago ${ }^{1,8,10}$. La presión esofágica representa la presión pleural y la presión gástrica refleja la presión abdominal. La Pdi es la diferencia entre la presión gástrica y esofágica durante maniobras de respiración tranquila o esfuerzo máximo, refleja la tensión desarrollada por el diafragma y depende del volumen pulmonar, por lo cual su medición debe ser estandarizada por el volumen pulmonar al cual se realiza la maniobra ${ }^{1,10}$. La medición de la Pdi permite estudiar específicamente la función del diafragma, mientras que la medición de la PIMax no permite discriminar entre los diferentes grupos musculares inspiratorios.

La estimulación eléctrica o magnética del nervio frénico permite evaluar la fuerza de los músculos respiratorios mediante maniobras independientes del esfuerzo del operador, permite medir las señales electromiográficas y la velocidad de conducción nerviosa, evaluar ambos hemidiafragmas por separado ${ }^{29-32}$. Los valores normales de Pdi mediante esta técnica fluctúan entre 8,8 y $33 \mathrm{~cm} \mathrm{H} \mathrm{H}_{2} \mathrm{O}$. Debido al amplio rango de valores normales, esta técnica sería útil principalmente en pacientes con debilidad muscular severa. La ejecución de esta técnica requiere personal altamente entrenado, la estimulación eléctrica a nivel cervical puede ser dolorosa, el fenómeno de potenciación puede sobrestimar la fuerza muscular, puede ser difícil localizar el nervio frénico en sujetos obesos o con malformaciones cervicales, es riesgoso en niños pequeños y estaría contraindicada en pacientes epilépticos ${ }^{29-32}$.

En resumen, la evaluación sistemática de los pacientes con debilidad de los músculos respiratorios estaría basada en los siguientes elementos: a) Historia clínica y examen físico; b) Radiografía, ultrasonografía y fluoroscopia de tórax; c) Pruebas de función pulmonar (volúmenes y capacidades pulmonares, $\mathrm{DL}_{\mathrm{CO}}$, gases arteriales); d) Medición de la fuerza máxima de los músculos respiratorios (PIMax, PEMax); e) Medición de la presión transdiafragmática (Pdi); f) Medición de la presión nasal máxima (SNIP); g) Estimulación eléctrica o magnética de los nervios frénicos.

\section{Bibliografía}

1.- GREEN M, MOXHAM J. Respiratory muscle in health and disease. In: Barnes P, ed, Respiratory medicine: recent advances. Oxford: Butterworth-Heinemann, 1993; pp 252-75.

2.- MURCIANO D, RIGAUD D, PINGLETON S, ARMENGAUD M H, MELCHIOR J C, AUBIER M. Diaphragmatic function in severely malnourished patients with anorexia nervosa. Effects of renutrition. Am J Respir Crit Care Med 1994; 150: 1569-74.

3.- ROCHESTER D F, BRAUN N M. Determinants of maximal inspiratory pressure in chronic obstructive pulmonary disease. Am Rev Respir Dis 1985; 132: 42-7.

4.- SIMILOWSKI T, DERENNE J P. Inspiratory muscle 
testing in stable COPD patients. Eur Respir J 1994; 7 : 1871-6.

5.- MCPARLAND C, KRISHNAN B, WANG Y, GALLAGHER C G. Inspiratory muscle weakness and dyspnea in chronic heart failure. Am Rev Respir Dis 1992; 146: 467-72.

6.- COAKLEY J H, NAGENDRAN K, HONAVAR M, HINDS C J. Preliminary observations on the neuromuscular abnormalities in patients with organ failure and sepsis. Intensive Care Med 1993; 19: 323-8.

7.- CELLI B R. Respiratory management of diaphragm paralysis. Semin Respir Crit Care Med 2002; 23: 275 81.

8.- MAISH M S. The diaphragm. Surg Clin North Am 2010; 90: 955-68.

9.- MOXHAM J. Respiratory muscle fatigue: mechanisms, evaluation and therapy. Br J Anaesth 1990; 65: 43-53.

10.- MIER-JEDRZEJOWICZ A, BROPHY C, MOXHAM J, GREEN M. Assessment of diaphragm weakness. Am Rev Respir Dis 1988; 137: 877-83.

11.- WHITE J E, DRINNAN M J, SMITHSON A J, GRIFFITHS C J, GIBSON G J. Respiratory muscle activity and oxygenation during sleep in patients with muscle weakness. Eur Respir J 1995; 8: 807-14.

12.- ALEXANDER C. Diaphragmatic movements and the diagnosis of diaphragmatic paralysis. Clin Radiol 1966; 17: 79-83.

13.- HOUSTON J G, ANGUS R M, COWAN M D, MCMILLAN N C, THOMSON N C. Ultrasound assessment of normal hemidiaphragmatic movement: relation to inspiratory volume. Thorax 1994; 49: 500-3.

14.- LEROLLE N, DIEHL J L. Ultrasonographic evaluation of diaphragmatic function. Crit Care Med 2011; 39: 2760-1.

15.- POLKEY M I, GREEN M, MOXHAM J. Measurement of respiratory muscle strength. Thorax 1995; 50: 1131-5.

16.- LAROCHE C M, CARROLL N, MOXHAM J, GREEN M. Clinical significance of severe isolated diaphragm weakness. Am Rev Respir Dis 1988; 138: 862-6.

17.- BRAUN N M, ARORA N S, ROCHESTER D F. Respiratory muscle and pulmonary function in polymyositis and other proximal myopathies. Thorax 1983; 38: 61623.

18.- BLACK L F, HYATT R E. Maximal respiratory pressures: normal values and relationship to age and sex. Am Rev Respir Dis 1969; 99: 696-702.

19.- LEECH J A, GHEZZO H, STEVENS D, BECKLAKE M R. Respiratory pressures and function in young adults. Am Rev Respir Dis 1983; 128: 17-23.

20.- BRUSCHI C, CERVERI I, ZOIA M C, FANFULLA F, FIORENTINI M, CASALI L, et al. Reference values of maximal respiratory mouth pressures: a populationbased study. Am Rev Respir Dis 1992; 146: 790-3.

21.- KOULOURIS N, MULVEY D A, LAROCHE C M, GREEN M, MOXHAM J. Comparison of two different mouthpieces for the measurement of PImax and PEmax in normal and weak subjects. Eur Respir J 1988; 1: 8637.

22.- HAMNEGARD C H, WRAGG S, KYROUSSIS D, AQUILINA R, MOXHAM J, GREEN M. Portable measurement of maximum mouth pressures. Eur Respir J 1994; 7: 398-401.

23.- SMYTH R J, CHAPMAN K R, REBUCK A S. Maximal inspiratory and expiratory pressures in adolescents. Normal values. Chest 1984; 86: 568-72.

24.- HERITIER F, RAHM F, PASCHE P, FITTING J W. Sniff nasal inspiratory pressure. A noninvasive assessment of inspiratory muscle strength. Am J Respir Crit Care Med 1994; 150: 1678-83.

25.- KOULOURIS N, MULVEY D A, LAROCHE C M, SAWICKA E H, GREEN M, MOXHAM J. The measurement of inspiratory muscle strength by sniff esophageal, nasopharyngeal, and mouth pressures. Am Rev Respir Dis 1989; 139: 641-6.

26.- KYROUSSIS D, MILLS G, HAMNEGARD C H, WRAGG S, ROAD J, GREEN M, et al. Inspiratory muscle relaxation rate assessed from sniff nasal pressure. Thorax 1994; 49: 1127-33.

27.- ULDRY C, FITTING J W. Maximal values of sniff nasal inspiratory pressure in healthy subjects. Thorax 1995; 50: 371-5.

28.- MULVEY D A, ELliotT M W, KOULOURIS N G, CARROLL M P, MOXHAM J, GREEN M. Sniff esophageal and nasopharyngeal pressures and maximal relaxation rates in patients with respiratory dysfunction. Am Rev Respir Dis 1991; 143: 950-3.

29.- MIER A, BROPHY C, MOXHAM J, GREEN M. Phrenic nerve stimulation in normal subjects and in patients with diaphragmatic weakness. Thorax 1987; 42: 885-8.

30.- MIER A, BROPHY C, MOXHAM J, GREEN M. Twitch pressures in the assessment of diaphragm weakness. Thorax 1989; 44: 990-6.

31.- SIMILOWSKI T, FLEURY B, LAUNOIS S, CATHALA H P, BOUCHE P, DERENNE J P. Cervical magnetic stimulation: a new painless method for bilateral phrenic nerve stimulation in conscious humans. J Appl Physiol 1989; 67: 1311-8.

32.- WRAGG S, AQUILINA R, MORAN J, RIDDING M, HAMNEGARD C, FEARN T, et al. Comparison of cervical magnetic stimulation and bilateral percutaneous electrical stimulation of the phrenic nerves in normal subjects. Eur Respir J 1994; 7: 1788-92.

Correspondencia a:

Dr. Fernando Saldías Peñafiel

Departamento de Enfermedades Respiratorias

Pontificia Universidad Católica de Chile.

Marcoleta 350-1er Piso

Santiago, Chile.

Email: fsaldias@med.puc.cl 